\title{
ESCALAS TEMPORAIS DA VARIABILIDADE PLUVIOMÉTRICA NA BACIA HIDROGRÁFICA DO RIO MUNDAÚ
}

\author{
DJANE FONSECA DA SILVA ${ }^{1}$, FRANCISCO DE ASSIS SALVIANO DE SOUSA ${ }^{2}$, MARY TOSHIE \\ KAYANO $^{3}$ \\ ${ }^{1}$ Universidade Federal do Ceará (UFC/Campus Cariri), Juazeiro do Norte, CE \\ ${ }^{2}$ Universidade Federal de Campina Grande/Departamento de Ciências Atmosféricas (UFCG/DCA), \\ Campina Grande, PB \\ ${ }^{3}$ Instituto Nacional de Pesquisas Espaciais/Centro de Previsão de Tempo e Estudos Climáticos \\ (INPE/CPTEC), São José dos Campos, SP
}

djane.fonseca@cariri.ufc.br, fassis@dca.ufcg.edu.br, mary.kayano@cptec.inpe.br

Recebido Julho 2009 - Aceito Fevereiro 2010

\begin{abstract}
RESUMO
Foram utilizados dados de precipitação da bacia hidrográfica do rio Mundaú, nos Estados de Alagoas e Pernambuco, obtidos da ANA, de 1955 a 1991. As análises foram feitas para três regiões desta bacia: Alto Mundaú (AM), Médio Mundaú (MM) e Baixo Mundaú (BM). Análises do Índice de anomalia de chuva (IAC) anual mostraram que as séries destas regiões têm variações temporais distintas, exceto pela dominância de anos secos no início do período de análise. Portanto, em alguns anos os fenômenos de grande escala podem ter o mesmo efeito nas três regiões da bacia hidrográfica do rio Mundaú e não em outros. Para as Análises de Ondaletas (AO), foi usado um índice de precipitação para cada região calculado pela média das séries de anomalias normalizadas das estações. Os resultados mostraram que a variabilidade de precipitação é definida por multi-escalas temporais localizadas em certos intervalos de tempo. No entanto, a variabilidade interanual do El Niño/Oscilação Sul (ENOS) e a variabilidade decenal influenciaram a variabilidade pluviométrica local. A partir desses resultados espera-se que a bacia hidrográfica tenha mais informações para a gestão de seus recursos hídricos e monitoramento climatológico.
\end{abstract}

Palavras-chave: Análise de Ondaleta, Oscilação Decenal do Pacífico, ENOS, Índice de Anomalia de Chuva.

ABSTRACT: TEMPORAL SCALES OF THE PLUVIOMETRIC VARIABILITY ON THE MUNDAÚ (AL and PE) RIVER BASIN: The Rain Anomaly Index (RAI) and the Wavelet Analysis (WA) were used to study the rainfall variability in different regions of the Mundaú river basin. ANA (Water National Agency) monthly rainfall data over the basin (States of Alagoas and Pernambuco) for the period 1955 to 1991 were used. The analysis were performed for three regions of this basin: High Mundaú (HM), Medium Mundaú (MM) and Low Mundaú (LM). Analyses of the RAI annual series show distinct temporal variations among these regions, except for the predominance of dry years during the beginning of the analysis period. Therefore, in some years the large scale phenomena might have the same effect on the three regions of the Mundaú basin, and have not on another. For WA and for each region a precipitation index calculated as the average of the normalized anomaly series of the stations was used. The results showed that the rainfall variability is defined by temporal multi-scales localized in certain time intervals. However, the ENSO related interannual variability and the decadal variability influenced the local rainfall variability. From these results, it is aimed that the Mundaú river basin would have more information for the management of its water resources and climate monitoring.

Keywords: Wavelet Analysis, Pacific Decadal Oscillation, ENSO, Rainfall Anomaly Index. 


\section{INTRODUÇÃO}

O nordeste do Brasil (NEB) é conhecido por apresentar grande variabilidade da precipitação, ou seja, anos com secas severas ou com chuvas excessivas, que têm sido relacionadas aos padrões de anomalias de grande escala da circulação atmosférica global principalmente o fenômeno El-Niño-Oscilação Sul (ENOS).

As secas severas no NEB, objeto de inúmeros estudos por seus impactos sociais e econômicos, têm sido relacionadas à ocorrência do El Niño. Entretanto, a relação entre El Niño e as secas no NEB não é unívoca (Kane e Trivedi, 1988; Kane, 1992; Kane, 1997). Por exemplo, Kane (1997) mostrou que dos 46 El Niño (fortes e moderados) do período de 1849-1992, somente $21(45 \%)$ estiveram associados a secas severas em Fortaleza. Este autor usou somente Fortaleza em vista de que Kane and Trivedi (1988) mostraram que a precipitação nesta localidade se correlaciona positiva e significativamente com as estações do NEB ao norte de $10^{\circ} \mathrm{S}$.

As ocorrências simultâneas de El Niño e secas no NEB se limitam a alguns eventos específicos. Isso se deve ao fato de que a precipitação nessa região é também fortemente relacionada às anomalias de temperatura da superfície do mar (ATSMs) do Atlântico tropical. Moura e Shukla (1981) mostraram que para alguns anos, os eventos de secas do NEB estão associados a um dipolo meridional de ATSMs no Atlântico tropical. A posição e intensidade da Zona de Convergência Intertropical (ZCIT) são afetadas e, por sua vez, influencia a precipitação no NEB. $\mathrm{O}$ modo de dipolo envolve variações de TSM em ambos os hemisférios, em escalas de tempo sazonal, interanual e decenal (Servain, 1991). Kayano e Andreoli (2006) mostraram que a variabilidade de precipitação no NEB está mais fortemente relacionada com as condições do Atlântico tropical, do que com as do Pacífico tropical.

Vale lembrar que apesar da variabilidade de TSM no Pacífico ser dominada pela variabilidade interanual associada ao ENOS, outros fenômenos de escala de tempo mais longa também como a Oscilação Decenal do Pacífico (ODP) também atuam neste setor. Zhang et al. (1997) separaram a variabilidade temporal do Pacífico em duas componentes: uma relacionada à escala de variabilidade interanual do ciclo ENOS, e a outra linearmente independente incluindo toda a variabilidade interdecenal. Além disso, os espectros das séries de ATSM, para os Oceanos Atlântico e Pacífico, são caracterizados por oscilações em escalas de tempo interanual e decenal, sendo a escala interanual mais pronunciada na região do Pacífico, e a decenal no Atlântico (Hastenrath e Kaczmarczyk, 1981; Sperber e Hameed, 1993; Mehta e Delworth, 1995).

Os efeitos combinados da ODP e do ENOS na precipitação da América do Sul foram estudados por Andreoli e Kayano (2005), Kayano e Andreoli (2007) e Kayano et al (2009). Estes trabalhos mostram que os efeitos do ENOS na precipitação da América do Sul podem ser acentuados ou reduzidos, dependendo se o ENOS e a ODP estão na mesma fase ou em fases opostas. De forma independente, as multi-escalas da variabilidade temporal da série da precipitação de Fortaleza foram analisadas (Markham, 1974; Chu, 1984; Hastenrath e Kaczmarczyk 1981; Andreoli et al. 2004).

No entanto, para a bacia hidrográfica do rio Mundaú, que enfrenta em alguns anos a problemática da escassez hídrica e que abastece municípios importantes do NEB, esse tipo de estudo ainda não foi realizado. Deste modo, um trabalho mais detalhado desta bacia hidrográfica reveste-se de importância quando analisados os impactos do clima em áreas de interesse sócio-econômico e ambiental. É através de estudos como o presente, que se torna possível conhecer os problemas da região e ao mesmo tempo tentar solucionálos ou minimizá-los por meio de planejamento de ações nos setores social, econômico e ambiental. Especificamente, na bacia hidrográfica do rio Mundaú, o presente estudo será muito útil para o desenvolvimento regional através da gestão dos recursos naturais locais, pois este trabalho visa ser uma ferramenta em tal ação. A importância da pesquisa também está no fato de que esse rio abastece a região metropolitana da cidade de Maceió. Somando-se à compreensão dos mecanismos geradores de precipitação, da sua variabilidade espaço-temporal e dos seus efeitos que, ora afetam as atividades agrícolas e consequentemente a população da região, a sociedade ganhará no sentido de planejar suas atividades e reduzir perdas.

Para tanto, as variações da precipitação em regiões da bacia hidrográfica do rio Mundaú serão estudadas utilizando a Análise de Ondaleta (AO), que é uma técnica apropriada para determinar as escalas de variabilidade dominantes em uma série temporal, bem como suas localizações no tempo. Nos últimos anos esta técnica tem sido bastante usada em meteorologia, por exemplo, nos trabalhos de Weng e Lau (1994), Torrence e Webster (1999), Abreu Sá et al. (1998), Vitorino (2003), Tucci e Braga (2003), Schneider et al. (2005), Andreoli et al. (2004), Andreoli e Kayano (2004), Labat et al. (2005), Da Silva (2009), dentre outros. Sua aplicabilidade na análise de sinais se deve principalmente porque permite decompor uma série temporal em diferentes níveis de resolução tempo-frequência e, então determinar, as componentes da variabilidade dominante (Gu e Philander, 1995; Torrence e Compo, 1998; Barbosa et al., 2004 dentre outros).

Adicionalmente, o Índice de Anomalia de Chuva (IAC) para caracterização da variabilidade espaço-temporal da precipitação numa região, tem se mostrado eficaz em estudos para várias regiões do globo, incluindo o NEB. Freitas (2004; 
2005) calculou o IAC de algumas localidades no Estado do Ceará, e fez comparações das condições atuais de precipitação com os valores históricos, bem como avaliou sua distribuição espacial. Mauget (2005) encontrou alta incidência de anos úmidos na América do Norte durante 1972 a 1998, com oito dos dez anos mais úmidos desde 1901, ou seja, oito eventos aconteceram durante esse último período de 27 anos. Gonçalves et al. (2006), através do IAC para 15 estações situadas no rio São Francisco, mostraram que a incidência de anos secos é substancialmente maior na região estudada e analisaram a ocorrência de cheias e inundações no sertão pernambucano. Segundo Repelli et al. (1998), o IAC parece ser apropriado para utilização em regiões semi-áridas e ou tropicais, especialmente para o NEB. Da Silva (2009) justificou que o IAC em relação ao índice Palmer Drought Severity Index-PDSI (Havens, 1969; Steila, 1971; Alley, 1984 e 1985; Guttman, 1991), é mais vantajoso porque o PDSI é calculado com base em dados de evapotranspiração, infiltração, escoamento superficial eventual, etc. O IAC tem a vantagem de necessitar somente de dados de precipitação.

Do acima exposto, o objetivo do presente trabalho é usar um índice simples como o IAC e através da AO estudar a variabilidade pluviométrica de três regiões da bacia hidrográfica do rio Mundaú, examinando aspectos da variabilidade sazonal e interanual, bem como determinando as escalas temporais dominantes. Esta bacia localiza-se nos Estados de Alagoas e Pernambuco. As diferenças e similaridades entre as regiões são também analisadas.

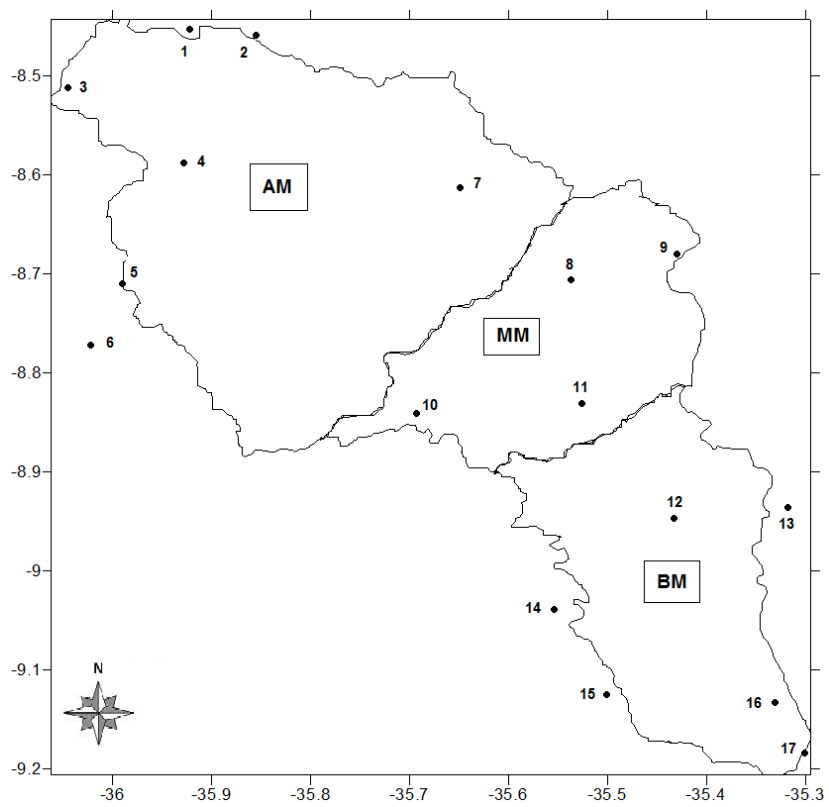

Figura 1 - Bacia hidrográfica do rio Mundaú e 17 localidades usadas neste trabalho.

\section{DADOS E METODOLOGIA}

\section{1 Área de Estudo}

A bacia hidrográfica do rio Mundaú, com uma área total de $4.126 \mathrm{~km}^{2}$ entre as latitudes de $9,4^{\circ}$ a $8,4^{\circ} \mathrm{S}$ e longitudes $35,4^{\circ}$ e $36,2^{\circ} \mathrm{W}$ (Figura 1) se estende sobre a parte noroeste de Pernambuco e centro-norte-oriental de Alagoas. O rio Mundaú nasce a oeste de Garanhuns, na parte sul do Planalto da Borborema e entra em Alagoas pela cachoeira da Escada, ao sul de Correntes, e a noroeste de Santana do Mundaú. Atravessa a área central da Mata Alagoana e chega ao litoral, com a sua foz afogada formando a Lagoa Mundaú (Tenório, 1985).

O período chuvoso da região, de fevereiro a julho, totaliza aproximadamente $72,6 \%$ do total médio anual de precipitação. Tal período coincide com a época em que esta região está sujeita às atuações de distúrbios de leste, que juntamente com os sistemas de escala local intensificam as chuvas, principalmente à noite nesta área (Costa et al., 2005).

\subsection{Dados Utilizados}

Os dados de precipitação utilizados foram obtidos através da Agência Nacional das Águas (ANA) no sítio www. ana.gov.br/hidroweb. Devido à disponibilidade de dados, utilizaram-se os seguintes 17 postos: Garanhuns, Caetés, Jucati, Jupi, Canhotinho, Bom Conselho, Brejão, Santana do Mundaú, São José da Lage, Ibateguara, União dos Palmares, Atalaia, Capela, Maceió, Rio Largo, Murici e Flexeiras. As localizações geográficas, e suas distribuições nas regiões do Alto Mundaú (AM), Médio Mundaú (MM) e Baixo Mundaú (BM) podem ser visualizadas na Tabela 1 e Figura 1. O período de estudo foi de 1955-1991 para as análises climatológicas do IAC e AO.

\subsection{Análise da Variabilidade Espaço-Temporal da Precipitação Local}

\section{I) Î́ndice de Anomalia de Chuva (IAC)}

A partir da metodologia de Rooy (1965), adaptada por Freitas $(2004 ; 2005)$ foram construídas as séries de IAC de 1955 a 1991 através de:

$$
\begin{aligned}
& I A C=3\left[\frac{(N-\bar{N})}{(\bar{M}-\bar{N})}\right], \text { para anomalias positivas; } \\
& I A C=-3\left[\frac{(N-\bar{N})}{(\bar{X}-\bar{N})}\right], \text { para anomalias negativas, }
\end{aligned}
$$


Sendo: $\mathrm{N}=$ precipitação média anual do ano que será gerado o IAC (mm); $\overline{\mathrm{N}}=$ precipitação média da série histórica $(\mathrm{mm}) ; \overline{\mathrm{M}}=$ média das dez maiores precipitações da série histórica (mm); $\overline{\mathrm{X}}=$ média das dez menores precipitações da série histórica $(\mathrm{mm})$; e anomalias positivas são valores acima da média e negativas, abaixo da média.

A análise do IAC permite classificar os anos como secos ou chuvosos, bem como analisar a frequência de suas ocorrências.

\section{II) Análise de Ondaletas}

A ondaleta de Morlet usada neste trabalho é uma exponencial complexa modulada por uma Gaussiana, $e^{i \omega_{o} \eta} e^{-\eta^{2} / 2}$, com $\eta=t / s$, onde $t$ é o tempo, $s$ é a escala da ondaleta e $\omega_{0}$ é uma freqüência não dimensional, que tem valor 6 para o caso da ondaleta de Morlet. O procedimento computacional da $\mathrm{AO}$ usada aqui é a descrita por Torrence e Compo (1998). A função de ondaleta de cada escala $s$ é normalizada por $s^{-1 / 2}$ para se obter energia unitária. A ondaleta de Morlet possui características semelhantes às de sinais meteorológicos com variação temporal suave.

Tabela 1 - Localidades utilizadas no estudo e suas respectivas coordenadas geográficas.

\begin{tabular}{cccc}
\hline Localidade & Latitude & Longitude & Região \\
\hline Garanhuns & $-8,883^{\circ}$ & $-36,483^{\circ}$ & $\mathrm{AM}$ \\
Caetés & $-8,783^{\circ}$ & $-36,633^{\circ}$ & $\mathrm{AM}$ \\
Jucati & $-8,70^{\circ}$ & $-36,45^{\circ}$ & $\mathrm{AM}$ \\
Jupi & $-8,70^{\circ}$ & $-36,416^{\circ}$ & $\mathrm{AM}$ \\
Canhotinho & $-8,866^{\circ}$ & $-36,20^{\circ}$ & $\mathrm{AM}$ \\
Bom Conselho & $-9,20^{\circ}$ & $-36,616^{\circ}$ & $\mathrm{AM}$ \\
Brejão & $-9,50^{\circ}$ & $-36,50^{\circ}$ & $\mathrm{AM}$ \\
Santana do Mundaú & $-9,166^{\circ}$ & $-36,216^{\circ}$ & $\mathrm{MM}$ \\
São José da Lage & $-9,004^{\circ}$ & $-36,05^{\circ}$ & $\mathrm{MM}$ \\
Ibateguara & $-8,983^{\circ}$ & $-35,933^{\circ}$ & $\mathrm{MM}$ \\
União dos Palmares & $-9,15^{\circ}$ & $-36,033^{\circ}$ & $\mathrm{MM}$ \\
Atalaia & $-9,50^{\circ}$ & $-36,0166^{\circ}$ & $\mathrm{BM}$ \\
Capela & $-9,30^{\circ}$ & $-36,133^{\circ}$ & $\mathrm{BM}$ \\
Maceió & $-9,666^{\circ}$ & $-35,70^{\circ}$ & $\mathrm{BM}$ \\
Rio Largo & $-9,466^{\circ}$ & $-35,85^{\circ}$ & $\mathrm{BM}$ \\
Murici & $-9,30^{\circ}$ & $-35,933^{\circ}$ & $\mathrm{BM}$ \\
Flexeiras & $-9,283^{\circ}$ & $-35,716^{\circ}$ & $\mathrm{BM}$ \\
\hline
\end{tabular}

O índice de precipitação de cada uma das três regiões foi calculado para todo o período de 1955 a 1991e se referem à média das estações de cada região de suas anomalias mensais de precipitação, normalizadas pelos respectivos desvios-padrão mensais, calculadas através de:

$$
\left.\operatorname{AVar}_{i, \mathrm{j}}=\left(\operatorname{Var}_{\mathrm{i}, \mathrm{j}}-\overline{\operatorname{Var}}\right) / \sigma_{\mathrm{i}} \overline{\operatorname{Var}}\right) / \sigma_{\mathrm{i}}
$$

Em que: AVar $r_{i, j}$ é a anomalia normalizada da precipitação no ano $\mathrm{j}=1,2,3, \ldots, \mathrm{N}$ e mês $\mathrm{i}=1,2,3, \ldots, 12 ; \operatorname{Var}_{i, j}$ é a precipitação no ano $\mathrm{j}=1,2,3, \ldots, \mathrm{N}$ e mês $\mathrm{i}=1,2,3, \ldots, 12$; $\overline{\operatorname{Var}}_{\imath} \overline{\operatorname{Var}}_{\imath}$ e $\sigma_{i} \sigma_{i}$ são a média climatológica e o desvio padrão do mês i.

O uso de um índice de precipitação é justificado pelo fato de que a variabilidade de precipitação na região NEB tem sinal homogêneo como mostrado anteriormente por diversos autores (Kousky e Chu 1978; Aceituno, 1988; Andreoli et al. 2004). Os índices de precipitação foram submetidos à $\mathrm{AO}$.

\section{RESULTADOS}

\section{1 Índice de Anomalia de Chuva: Variabilidade Interanual}

A Figura 2 mostra as médias anuais da precipitação nas três regiões do rio Mundaú para o período de 1955 a 1991. Neste período de análise, as regiões do MM e BM apresentam maiores valores da média anual de precipitação do que o AM. Comparando as três regiões, os maiores valores da média anual de precipitação ocorreram no BM até 1974 e após esse ano, no MM. De acordo com a SUDENE (1999), é nesta última região que ocorrem eventos de enchentes com mais frequência. Nota-se também, que os valores de precipitação média anual nas três regiões apresentam uma tendência de diminuição do ano de 1976 a 1983. Isso deve estar relacionado com o fato de o ano de 1983 ter sido extremamente seco.

Os menores valores das médias anuais no AM em relação às outras regiões, também podem ser observados a partir da climatologia das três regiões (Figura 3). Nesta figura observa-se também, que a quadra chuvosa se estende de abril a julho no MM e BM, enquanto no AM o período de chuva é mais ou menos homogêneo se estendendo de março a julho. Já os meses mais secos para o BM e MM ocorrem de outubro a janeiro, enquanto no AM, de setembro a dezembro.

Assim, a região do $\mathrm{AM}$, que tem o menor índice pluviométrico e período seco na primavera/verão, é uma região que necessita de uso de irrigação em períodos de escassez hídrica, devendo também ser observado o tipo de cultivo. Na região do $\mathrm{BM}$, devido aos maiores valores de precipitação, a agricultura é mais beneficiada, com o uso da irrigação em 


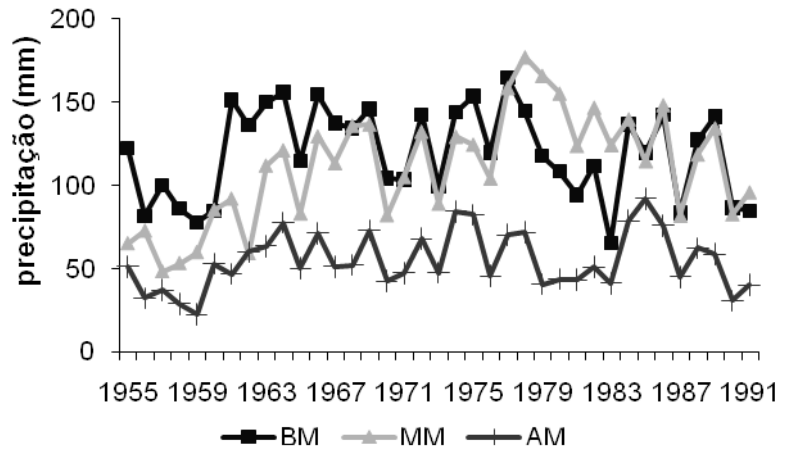

Figura 2 - Precipitações médias anuais de 1955 a 1991 para as três regiões.

períodos mais curtos do ano. Já no MM, atenta-se para o fato da região apresentar histórico de enchentes, o que pode prejudicar sócio-economicamente as plantações e os pequenos produtores. $\mathrm{O}$ mesmo não ocorre no $\mathrm{BM}$ tão frequentemente, devido sua topografia e a localização da foz ser em um estuário, o que em algumas situações facilita a descarga excedente do rio no Oceano Atlântico.

As Figuras 4, 5 e 6 mostram o IAC médio anual do $\mathrm{BM}, \mathrm{MM}$ e AM, respectivamente. Nestas figuras os valores negativos representam anos secos e os valores positivos, os anos chuvosos. No BM, ocorreram 18 anos chuvosos e 19 anos secos, que se distribuíram com dominância de anos chuvosos no período de 1961 a 1978. De fato, neste período 13 anos foram secos. Por outro lado, os anos secos ocorreram dominantemente nos períodos de 1956-1960 e de 1979-1991.

$\mathrm{Na}$ Figura 5 visualiza-se o IAC anual do MM, onde dos 37 anos da série, 21 anos foram úmidos e 16 anos foram secos, fato que reforça as conclusões da SUDENE (1999) no que se refere a freqüência de inundações desta região. Neste caso, nota-se uma seqüência de anos secos no período de 1955-1962, alternância de anos secos e chuvosos nos período de 1963-1976 e de 19871991, e uma seqüência de anos chuvosos no período de 1977-1988. Nessa região é também aparente um ponto de inversão na série de precipitação em 1974, com mais anos úmidos após esse ano. Essa "peculiaridade temporal" com um período de IACs positivos e outro, com IACs negativos, corrobora os resultados encontrados por Mauget (2005) para outras regiões continentais.

Na Figura 6 observa-se o IAC anual do AM de 1955 a 1991, no qual são contabilizados 22 anos secos e 15 anos úmidos. Dos 22 anos secos, alguns ocorreram em seqüência nos períodos de 1955-1961 1979-1983. Os demais anos secos se alternaram com os anos chuvosos.

Exceto pela sequência de anos secos no início de cada série, as séries de IAC anual das três regiões mostram variações temporais distintas.

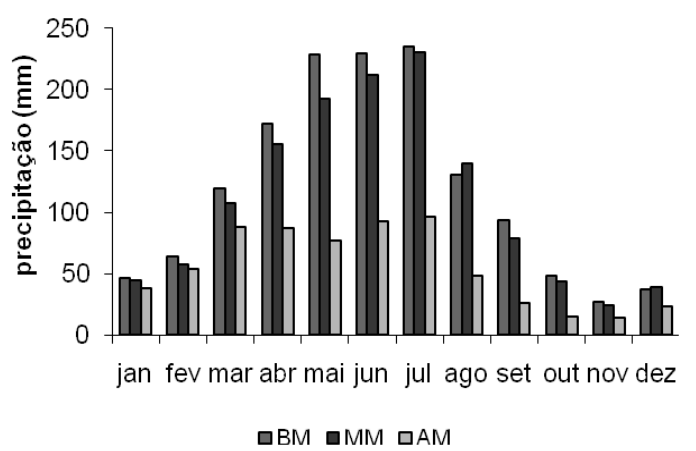

Figura 3 - Médias Climatológicas para as três regiões.

\subsection{Análises de Ondaleta: Variabilidade Decenal}

O pico dominante para o $\mathrm{BM}$, ao longo da série de 1955-1991, é notado na escala de 15,5 anos (Figura 7c). Picos estatisticamente não significativos são notados na escala de 5,5-7 anos e de aproximadamente 2 anos (Figura 7c). O pico significativo da escala de 15,5 anos resulta de altos valores do Espectro de Potência da Ondaleta (EPO) (Figura 7b), em todo o período de estudo, possivelmente ligados à variabilidade decenal. Os picos nas escalas de 2 anos e de 5,5-7 anos estão associados aos sinais do ENOS e são explicados pelos altos valores do EPO de 1963-1969 e de 1982-1986 (Figuras 7a e 7b).

Para o MM, o pico dominante ocorre na escala de 18,5 anos que é devido a altos valores de EPO em quase todo o período de análise (Figuras $8 \mathrm{~b}$ e $8 \mathrm{c}$ ). Um pico secundário (não significativo) ocorre nas escalas de $5-7,7$ anos, que é devido aos altos valores de EPO no período de 1960-1980 e de 1985-1991. Nas escalas interanual curta (em torno de 1 ou até menor) notam-se variâncias significativas resultantes dos eventos de 1963, 1978 e 1991 (Figuras 8a e Figura 8b). Esses anos apresentaram altos valores no índice Prp. Portanto a variabilidade na escala interanual curta (Figura $8 b$ ) também contribui para os totais pluviométricos.

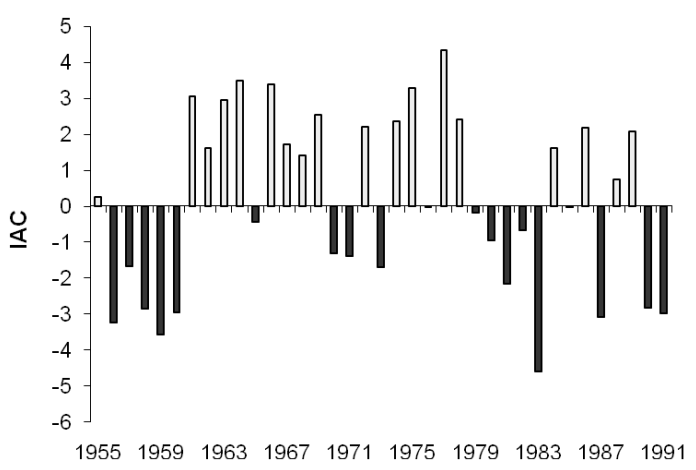

Figura 4 - IAC anual para o Baixo Mundaú. 


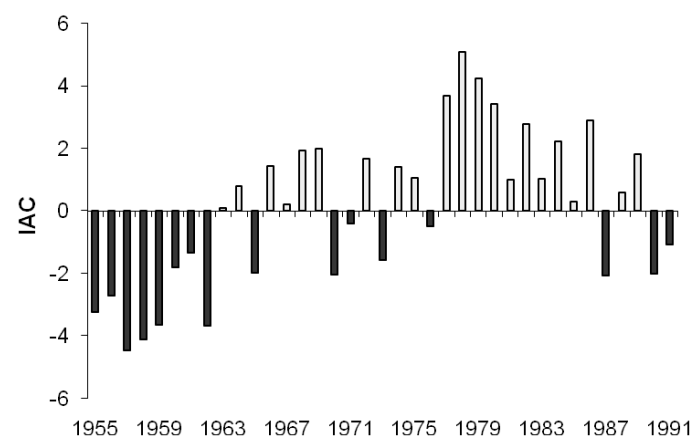

Figura 5 - IAC anual para o Médio Mundaú.

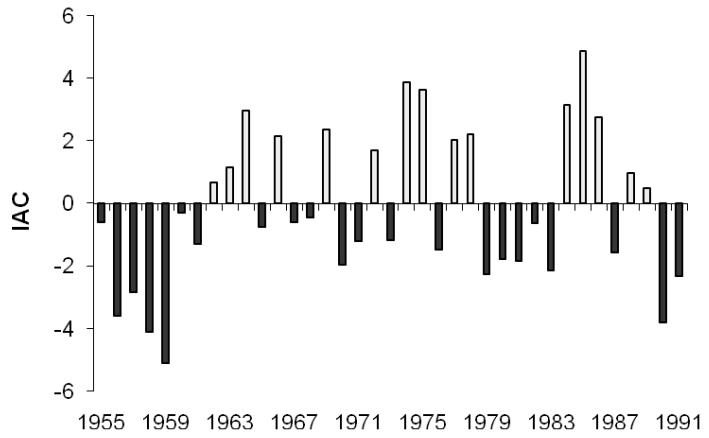

Figura 6 - IAC anual para o Alto Mundaú.

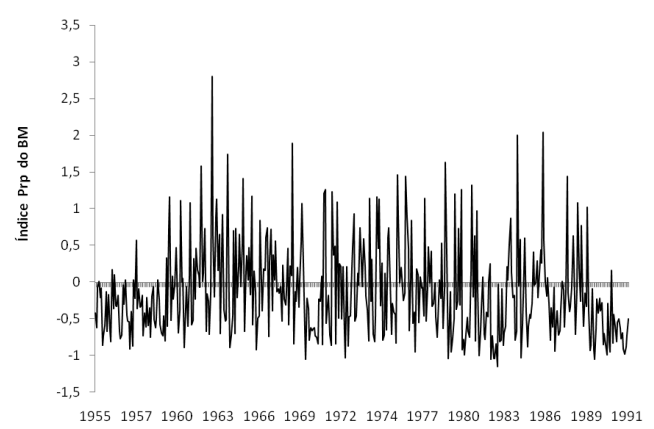

(a)

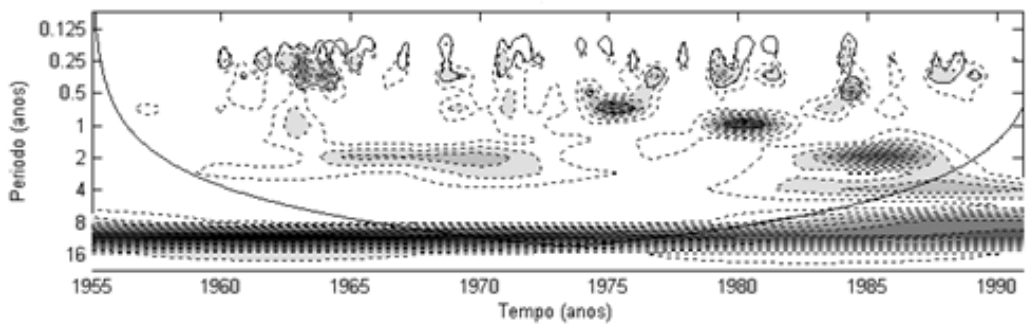

(b)

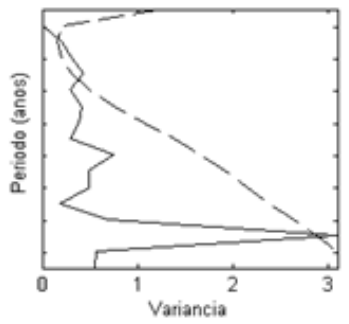

(c)

Figura 7 - a) Índice de precipitação para BM (Prp) normalizado pelo desvio padrão; b) Espectro de potência de ondaleta (EPO) para Prp. Contornos sombreados correspondem a variâncias normalizadas significativas ao nível de 5\%. A curva em forma de U representa o cone de influência, sob a qual o efeito de borda é importante; c) Espectro de potência global (EPG), com o contorno tracejado indicando que o EPG é significativo ao nível de confiança de $95 \%$.

Para as três regiões da bacia hidrográfica, as variações em escalas interanual curta e interanual contribuíram positivamente para as precipitações locais. Esses resultados indicam que a variabilidade de precipitação na bacia é definida por multiescalas temporais localizadas em certos intervalos de tempo.

Principalmente a variabilidade interanual ligada ao ciclo de ENOS e a variabilidade decenal, que foi dominante nas três sub-bacias, influenciam na variabilidade pluviométrica local. Esses resultados encontram-se de acordo com os observados por Markham (1974), Chu (1984) e Hastenrath e Kaczmarczyk (1981), Andreoli et al. (2004), todos realizados para Fortaleza, também no NEB.

Diante do fato de que os eventos nas escalas temporais interanuais e decenais contribuem para a variabilidade da precipitação nas três regiões da Bacia do rio Mundaú, o monitoramento destes fenômenos é importante para inferir as condições climáticas nestas regiões. E somente através de um monitoramento sistemático destes fenômenos, bem como das condições pluviométricas da região, é que se pode maximizar o aproveitamento de água de chuva na gestão agrícola, pesqueira, social e energética, por exemplo.

\section{CONCLUSÕES}

Este trabalho usou os dados mensais de precipitação de 17 estações da bacia hidrográfica do rio Mundaú do período de 1955-1991 e analisou os aspectos sazonais, interanuais e da variabilidade de mais longo prazo desta variável em três regiões 


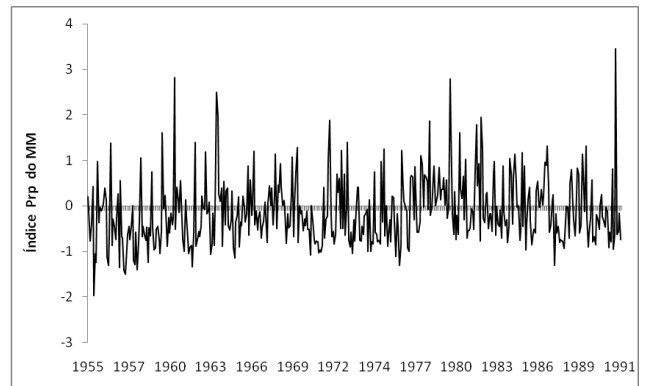

(a)

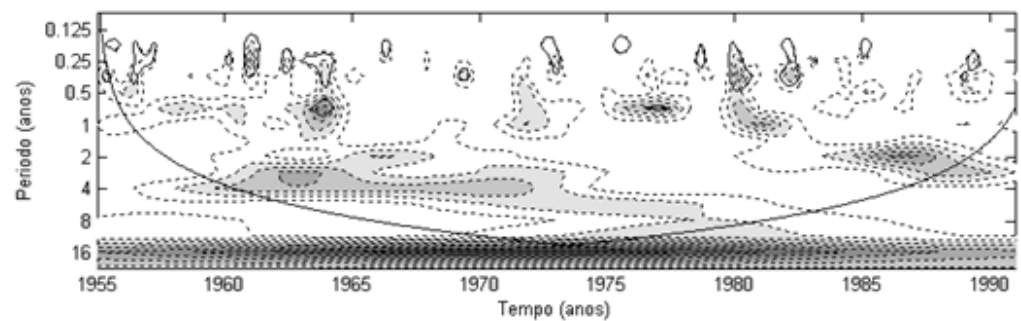

(b)

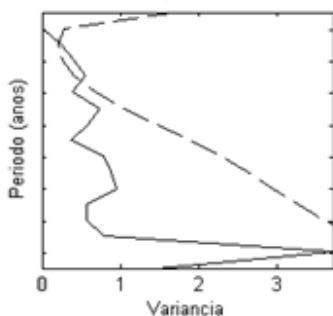

(c)

Figura 8 - a) Índice de precipitação para MM (Prp) normalizado pelo desvio padrão; b) Espectro de potência de ondaleta (EPO) para Prp. Contornos sombreados correspondem a variância normalizadas significativas ao nível de $5 \%$. A curva em forma de U representa o cone de influência, sob a qual o efeito de borda é importante; c) Espectro de potência global (EPG), com o contorno tracejado indicando que o EPG é significativo ao nível de confiança de $95 \%$.

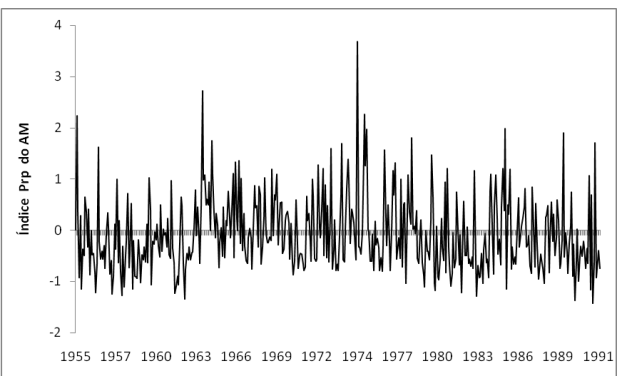

(a)

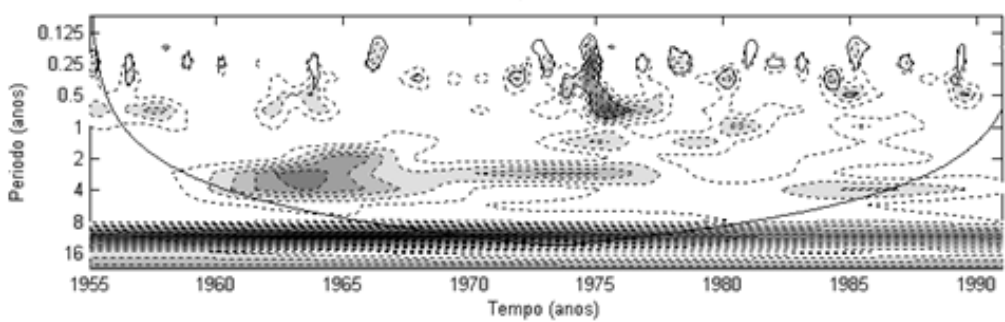

(b)

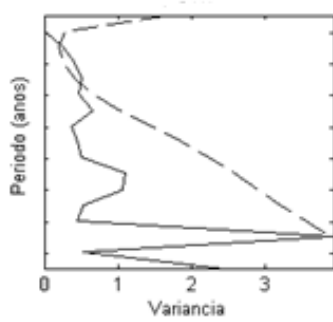

(c)

Figura 9 - Índice de precipitação para AM (Prp) normalizado pelo desvio padrão; b) Espectro de potência de ondaleta (EPO) para Prp. Contornos sombreados correspondem a variância normalizados significativas ao nível de 5\%. A curva em forma de U representa o cone de influência, sob a qual o efeito de borda é importante; c) Espectro de potência global (EPG), com o contorno tracejado indicando que o EPG é significativo ao nível de confiança de $95 \%$. 
(BM, MM e AM) desta bacia. Através dos valores médios anuais de precipitação foram notados os menores valores no $\mathrm{AM}$ em relação às outras regiões. O principal aspecto sazonal é que a quadra chuvosa se estende de abril a julho no MM e $\mathrm{BM}$, enquanto no AM o período chuvoso estende-se de março a julho. Já os meses mais secos para o BM e MM são de outubro a janeiro enquanto no AM, de setembro a dezembro. Por outro lado, exceto pela dominância de anos secos no início do período de análise, foi detectado, através do IAC anual, que as séries têm variações temporais distintas. Portanto, em alguns anos os fenômenos de grande escala podem ter o mesmo efeito nas três regiões da bacia do Mundaú, enquanto em outros os efeitos podem não ser o mesmo ou com igual intensidade.

Verificou-se para a bacia hidrográfica do rio Mundaú, através da análise de ondaletas, que principalmente a variabilidade interanual ligada ao ciclo de ENOS, a variabilidade decenal, bem como a variabilidade interanual curta, influenciam na variabilidade pluviométrica local.

\section{AGRADECIMENTOS}

Os autores agradecem aos dois revisores pelas valiosas sugestões que contribuíram para a melhoria do texto final. Agradecimentos também são extensivos ao $\mathrm{CNPq}$, setor CT-Hidro, pela concessão de bolsa de pesquisa durante o Doutorado da primeira autora.

\section{REFERÊNCIAS BIBILOGRÁFICAS}

ABREU SÁ, L.D.; SAMBATTI, S.B.M.; GALVÃO, G.P. Ondeleta de Morlet aplicada ao estudo da variabilidade do Nível do rio Paraguai em Ladário, MS; Número Especial, Pesquisa Agropecuária Brasileira, Brasília, vol.33, p.1775-1785, out. 1998.

ACEITUNO, P. On the functioning of the Southern Oscillation in the South American Sector. Part 1: surface climate, Monthly Weather Review, v.116, p.505-524, 1988.

ALLEY, W.M. The Palmer Drought Severity Index: Limitations and Assumptions, Journal of Climate and Applied Meteorology, v. 23, p.1100-1109, 1984.

ALLEY, W.M. The Palmer Drought Severity Index as a Measure of Hydrologic Drought, Water Resources Bulletin, 21 (1), p.105-114, 1985.

ANDREOLI, R.V.; KAYANO, M.T. Multi-scale variability of the sea surface temperature in the Tropical Atlantic, Journal of Geophysical Research, 109, C05009, 2004.

ANDREOLI, R.V.; KAYANO, M.T. Enso-Related Rainfall Anomalies in South America and Associated Circulation Features During Warm and Cold Pacific Decadal Oscillation Regimes, International Journal of Climatology.
Internacional Journal Climatology, 25: p. 2017-2030, 2005.

ANDREOLI, R.V., KAYANO, M.T., GUEDES, R.L., OYAMA, M.D., ALVES, M.A.S. A influência da temperatura da superfície do mar dos Oceanos Pacífico e Atlântico na variabilidade de precipitação em Fortaleza, Revista Brasileira de Meteorologia, v.19, n.3, p.337-344, 2004.

BARBOSA, E.B.M.; ROSA, M.M.; VIJAYKUMAR, N.L.; BOLZAN, M.J.A.; TOMASELLA, J. Caracterização por Ondeletas de Processos Físicos Não-Lineares na Microbacia Amazônica, INPE, São José dos Campos, 2004.

$\mathrm{CHU}, \mathrm{P}$. S. Time and space variability of rainfall and surface circulation in the northeast Brazil - Tropical Atlantic sector, Journal of Meteorological Society of Japan, v.26, n.2, p.363-369, 1984.

COSTA, M C.; OLIVEIRA, M. C. F.; MORAES, J. C.; BARRETO, P. N.; DANTAS, V. A.; CARVALHO, S. P. Comportamento e relação entre PRP e vazão na bacia do rio Mundaú, Alagoas e Pernambuco. XIV Congresso de Brasileiro de Agrometeorologia, Anais..., Campinas-SP, julho 2005.

DA SILVA, D. F. Análise de aspectos climatológicos, agroeconômicos, ambientais e de seus efeitos sobre a bacia hidrográfica do rio Mundaú (AL E PE), março de 2009, 212 p., Tese de Doutorado em Recursos Naturais, UFCG, Campina Grande (PB), 2009.

FREITAS, M. A. S. A Previsão de Secas e a Gestão Hidroenergética: OCaso da Bacia do Rio Parnaíba no Nordeste do Brasil. In: Seminário Internacional sobre Represas y Operación de Embalses, 2004, Puerto Iguazú. Anais do Seminário Internacional sobre Represas y Operación de Embalses. Puerto Iguazú : CACIER, 2004. v. 1. p. 1-1. FREITAS, M. A. S. Um Sistema de Suporte à Decisão para o Monitoramento de Secas Meteorológicas em Regiões Semi-Áridas. Revista Tecnologia, Fortaleza, v. Suplem, p. 84-95, 2005.

GONÇALVES, W.A.; CORREIA, M.F.; ARAÚJO, L.E.; DA SILVA, D.F.; ARAÚJO, H.A. Vulnerabilidade Climática do Nordeste Brasileiro: Uma análise de eventos extremos na Zona Semi-árida da bacia hidrográfica do São Francisco, XIV Congresso Brasileiro de Meteorologia, Anais...., Florianopólis, 2006.

GU, D., PHILANDER, S.G.H. Secular changes of annual and interannual variability in the tropics during the past century, Journal of Climate, vol. 8 (4), 864-876, 1995.

GUTTMAN, N.B. A Sensitivity Analysis of the Palmer Hydrologic Drought Index, Water Resources Bulletin, 27(5), p.797-807, 1991.

HASTENRATH, S., KACZMARCZYK, E. B. On spectra and coherence of tropical climate anomalies, Tellus, v.33, n.5, p.453-462, 1981. 
HAVENS, A.V. Economic Impact of Drought on Water Systems in Passaic River Basin, Journal New Brunswick, New Jersey Agricultural Experiment Station, 1969.

KANE, R. P. El Niño and La Niña events and rainfall in NE and South Brazil, Revista Brasileira de Geofísica., v.10, n.2, p.49-59, 1992.

KANE, R. P. Prediction of droughts in North-east Brazil: Role of ENSO and use of periodicities, International Journal of Climatology, v.17, p.655-665, 1997.

KANE, R. P.; TRIVEDI, N. B. Spectral characteristics of the annual rainfall series for northeast Brazil, Climate Change, v.13, p.317-336, 1988.

KAYANO, M. T; ANDREOLI R. V. Relationships between rainfall anomalies over northeastern Brazil and the El NiñoSouthern Oscillation. Journal of Geophysical Research, v.111, D13102, doi:10.1029/2005JD006142, 2006.

KAYANO, M. T; ANDREOLI R. V. Relations of South American summer rainfall interannual variations with the Pacific decadal oscillation. International Journal of Climatology, v.27, p.531-540, doi:10.1002/joc.1417, 2007.

KAYANO, M. T; OLIVEIRA C.P.; ANDREOLI R. V. Interannual relations between South American rainfall and tropical sea surface temperature anomalies before and after 1976. International Journal of Climatology, v.29, p.14391448, doi:10.1002/joc.1824, 2009.

KERR, R.A. A now dawn for sun-climate links? Science, Washington, DC, v.271, n5254, p.1360-1361, 1996.

KOUSKY, V.E.; CHU, P.S. Fluctuations in annual rainfall for northeast Brazil, Journal of Meteorological Society of Japan, v.56, p.457-466, 1978.

LABAT, D; RONCHAIL, J.; GUYOT, J.L. Recent advances in Wavelet analyses, part 2-Amazon, Parana, Orinoco and Congo discharges time scale variability. Journal of Hidrology, p.1-23, 2005.

MARKHAM, C. G. Apparent periodicities in rainfall at Fortaleza, Ceará, Brazil, Journal of Applied. Meteorology., v.13, p.176-179, 1974.

MAUGET, S.U.M. Índice Padronizado de Precipitação (SPI); C. Springer Science, 2005.

MEHTA,V.; DELWORTH, T. Decadal variability of the Tropical Atlantic ocean surface temperature in shipboard measurements and in a global ocean-atmosphere model, Journal of Climate, v.8, n.3, p.172-190, 1995.

MOURA, A.D.; SHUKLA J. On the dynamics of droughts in northeast Brazil: observations, theory and numerical experiments with a general circulation model, Journal of the. Atmospheric Sciences, v.38, p. 2653-2675, 1981.
REPELLI, C. A.; FERREIRA, N. S.; ALVES, J. M. B.; NOBRE, Carlos Afonso. Índice de anomalia de precipitação para o Estado do Ceará. In: X CONGRESSO BRASILEIRO DE METEOROLOGIA E VIII CONGRESSO DA FLISMET, 1998, Brasília DF. Anais do X Congresso Brasileiro de Meteorologia e VIII Congresso da FLISMET, 1998.

ROOY, M.P. VAN. A Rainfall Anomaly Index Independent of Time and Space, Notes, 14, 43, 1965.

SCHNEIDER M., VITORINO, M. I., SILVA DIAS, P.L. Monitoramento da Intrasazonalidade por meio da Transformada em Ondeletas, Simpósio Internacional de Climatologia, Anais..., Fortaleza-2005.

SERVAIN, J. Simple climate indice for the tropical Atlantic ocean and some applications. Journal of Geophysical Research, 96 (8):15137 - 15146, 1991.

SPERBER, K. R.; HAMEED, S. Phase Locking of Nordeste precipitation with sea surface temperatures, Geophysical Resource Letter, v.20, n.2, p.113-116, 1993.

STEILA, D. DroughtAnalysis in Four Southern States by a New Index, PhDDiss.,Athens, Ga., The University of Georgia, 1971.

SUDENE. Plano Diretor de Recursos Hídricos da Bacia do Rio Mundaú. Recife-PE, 1999.

TENÓRIO, R. S. Estudo Hidrometeorológico da Bacia do Rio Mundaú. Universidade Federal de Alagoas. MET. Maceió - Alagoas, Fevereiro, 1985.

TORRENCE, C.; COMPO, G.P.: A practical guide to wavelet analysis. Bulletin of the American Meteorological Society, v.79, p.61-78, 1998.

TORRENCE, C.; WEBSTER, P.J. : Interdecadal changes in the ENSO-monsoon system. J.Climate, 12, p.2679-2690, 1999.

TUCCI, C.E.M; BRAGA, B. Clima e Recursos Hídricos no Brasil, Coleção ABRH, 348 p., 2003.

VITORINO, M.I. Análise das Oscilações Intrasazonais sobre a América do Sul e Oceanos Adjacentes Utilizando a Análise de Ondeletas, 2003, 147 p. Tese de Doutorado em Meteorologia, INPE, São José dos Campos, 2003.

WENG, H. LAU, K-M. Wavelets, period doubling, and timefrequency localization with application to organization of convection over the Tropical Western Pacific. Journal of the Atmospheric Sciences, v.51, n.17, p.2523-2541, 1994.

ZHANG, Y.; WALLACE, J.M.; BATTISTI, D. ENSO-like interdecadal variability: 1900-93, Journal of Climate, v.10, p.1004-1020, 1997. 